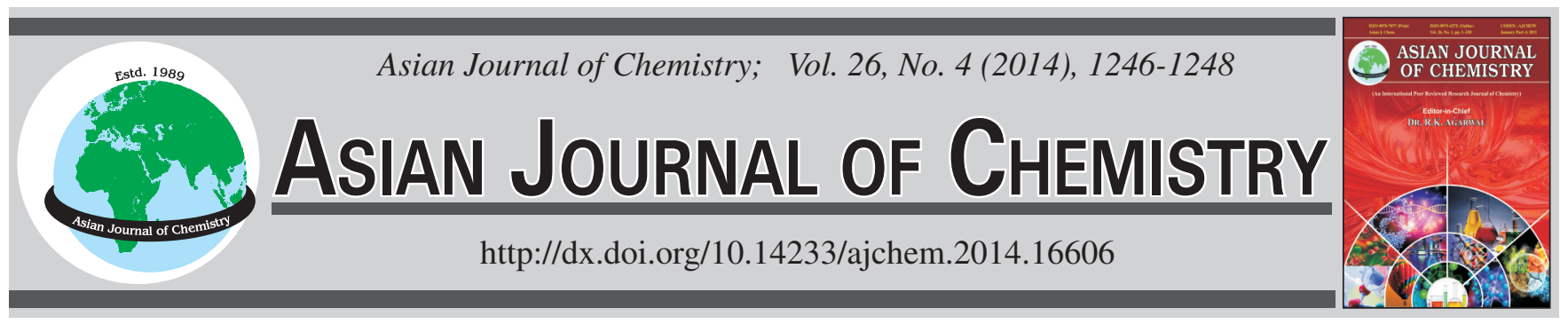

\title{
A Rapid, Convenient, Solventless Green Approach for the Synthesis of $\alpha$-Hydroxyphosphonates by Grinding
}

\author{
Du-Lin Kong ${ }^{1}$, Ren-Die Liư ${ }^{2}$, Guo-Zhu Li ${ }^{2}$, Peng-Wei Zhang ${ }^{1, *}$ and Ming-Shu Wu ${ }^{2}, *$
}

${ }^{1}$ School of Pharmaceutical Sciences, Hainan Medical University, Haikou 571199, Hainan Province, P.R. China

${ }^{2}$ College of Chemistry and Chemical Engineering, Hainan Normal University, Haikou 571158, Hainan Province, P.R. China

*Corresponding authors: Tel/Fax: +86 898 66893826, E-mail: zpw0803@163.com; wumingshu@126.com; 173548977@qq.com

An efficient and convenient approach to the condensation reaction of aromatic aldehydes and diethyl phosphite using $\mathrm{Na}_{2} \mathrm{CO}_{3}$ as catalyst with grinding at room temperature (without any solvent) is described. This method provides several advantages such as neutral condition, simple work-up procedure, high yields and reduced environmental impact.

Keywords: $\alpha$-Hydroxyphosphonate, Synthesis, Grinding.

\section{INTRODUCTION}

$\alpha$-Hydroxyphosphonate derivatives are important organophosphorus compounds associated with a wide variety of biological and pharmaceutical activities ${ }^{1}$. These derivatives are extensively used as synthetic intermediates ${ }^{2}$ with iterative manipulation of functional groups to $\alpha$-ketophosphonates ${ }^{3}$ and 1,2-diketones ${ }^{4}$. The hydrolyzed products of $\alpha$-hydroxyphosphonates exhibit a wide range of medicinal properties such as antibacterial $^{5}$, antiviral ${ }^{6}$ and anticancer activities ${ }^{7}$. A large number of methodologies for the synthesis of various $\alpha$-hydroxyphosphonates compounds have been extensively developed under various conditions. Recently, the synthesis of $\alpha$-hydroxyphosphonates with aldehydes and dialkyl (or) trialkyl phosphates using Lewis acids and bases catalysts such as $\mathrm{MgO}^{8}, \mathrm{NH}_{4} \mathrm{VO}_{3}{ }^{9}$, alumina $^{10}$, quinine ${ }^{11}$, lithium diisopropylamine $(\mathrm{LDA})^{12}, \mathrm{Ti}(\mathrm{OPri})_{2}{ }^{13}$, $\mathrm{LiClO}_{4} \cdot \mathrm{Et}_{2} \mathrm{O}, \mathrm{TMSCl}^{14}$, The majority of these processes suffer some drawbacks such as long reaction times, low yields of the products, requiring a stoichiometric amount of catalysts, costly metal ion and the use of highly toxic catalysts. Therefore, it is still required to develop a more efficient method particularly considering today's environmental concerns combined with economic aspects.

The grinding method is used more and more frequently in organic synthesis ${ }^{15}$. Compared with traditional methods, proposed method is more convenient and easily controlled. A great number of organic reactions can be carried out in higher yields, shorter times or milder conditions by the grinding method. It can even set off some reactions that cannot be carried out under traditional conditions. Herein we reported a simple procedure for the preparation of $\alpha$-hydroxyphosphonates catalyzed by $\mathrm{Na}_{2} \mathrm{CO}_{3}$ under grinding at room temperature. (Scheme-I).

\section{EXPERIMENTAL}

Melting points were uncorrected. Liquid aldehydes were distillated before use. NMR spectra were measured on Bruker Avance $400(400 \mathrm{MHz})$ spectrometer using TMS as internal reference and $\mathrm{CDCl}_{3}$ as solvent. IR spectra were determined as $\mathrm{KBr}$ pellets on Avatar 360 FT-IR spectrophotometer. Elemental analysis was carried out with a Yanaco Chncorder MT-3 analyzer.

General procedure: To $3 \mathrm{mmol}$ of aldehyde, $3 \mathrm{mmol}$ of diethylphosphite and anhydrous sodium carbonate $(3 \mathrm{mmol})$ were added and grinded at room temperature for $10 \mathrm{~min}$. After completion of the reaction, as indicated by thin-layer chromatography the reaction mixture was washed with water $(15 \mathrm{~mL})$ then the compounds were extracted with ethyl acetate. The organic layer were dried over anhydrous $\mathrm{Na}_{2} \mathrm{SO}_{4}$, filtered and concentrated in vacuo. Crystallization from acetone-pentane

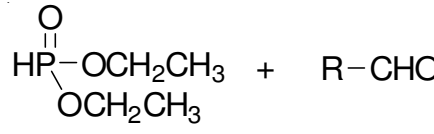

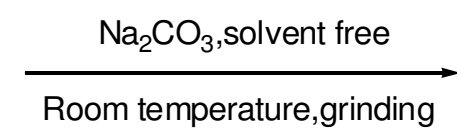

Scheme-I<smiles>[R]C(O)P(=O)(OCC)OCC</smiles> 
afforded products a-f white crystals (except for $\mathbf{d}$ and $\mathbf{f}$ which were orange crystals; Table-2).

Diethyl (hydroxyl)(phenyl)methylphosphonate (a): white crystals, Yield: $82 \%$, m.p. $83-85^{\circ} \mathrm{C} .{ }^{1} \mathrm{H}$ NMR $\left(\mathrm{CDCl}_{3}\right.$, $400 \mathrm{MHz}): \delta 7.36-7.18(\mathrm{~m}, 5 \mathrm{H}), 5.25(\mathrm{~s}, 1 \mathrm{H}), 4.64\left(\mathrm{~d},{ }^{2} J_{\mathrm{P}-\mathrm{H}}=\right.$ $10.7 \mathrm{~Hz}, 1 \mathrm{H}), 4.00-3.90(\mathrm{~m}, 4 \mathrm{H}), 1.26-1.17$ (m, 6H); ${ }^{13} \mathrm{C} \mathrm{NMR}$ $\left(\mathrm{CDCl}_{3}, 100 \mathrm{MHz}\right): \delta 147.5\left(\mathrm{~d},{ }^{2} J_{\mathrm{P}-\mathrm{C}}=3.0 \mathrm{~Hz}\right), 134.0,128.8$, $127.1,70.3\left(\mathrm{~d},{ }^{1} J_{\mathrm{P}-\mathrm{C}}=157.0 \mathrm{~Hz}\right), 62.9\left(\mathrm{~d},{ }^{2} J_{\mathrm{P}-\mathrm{C}}=7.1 \mathrm{~Hz}\right), 16.0$ $\left(\mathrm{d},{ }^{3} J_{\mathrm{P}-\mathrm{C}}=6.0 \mathrm{~Hz}\right) ; \mathrm{IR}\left(\mathrm{KBr}, \mathrm{v}_{\max }, \mathrm{cm}^{-1}\right): 3264$ (brs, OH), 1228 $(\mathrm{P}=\mathrm{O})$; Anal. calcd. for $\mathrm{C}_{11} \mathrm{H}_{17} \mathrm{O}_{4} \mathrm{P}: \mathrm{C}, 54.10$; $\mathrm{H}, 7.02$; Found: C, 53.95; H, 6.90 .

Diethyl (hydroxy)(p-tolyl)methylphosphonate (b): white crystals, Yield: $76 \%$, m.p. $98-100{ }^{\circ} \mathrm{C} .{ }^{1} \mathrm{H} \mathrm{NMR}\left(\mathrm{CDCl}_{3}\right.$, $400 \mathrm{MHz}): \delta 7.29-7.00(\mathrm{~m}, 4 \mathrm{H}), 5.26(\mathrm{~s}, 1 \mathrm{H}), 4.58\left(\mathrm{~d},{ }^{2} J_{\mathrm{P}-\mathrm{H}}=\right.$ $10.1 \mathrm{~Hz}, 1 \mathrm{H}), 4.21-4.02(\mathrm{~m}, 4 \mathrm{H}), 2.32$ (s, 3H), 1.27-1.19 (m, $6 \mathrm{H}) ;{ }^{13} \mathrm{C} \mathrm{NMR}\left(\mathrm{CDCl}_{3}, 100 \mathrm{MHz}\right): \delta 145.4,137.1,122.2,121.4$, $69.1\left(\mathrm{~d},{ }^{1} J_{\mathrm{P}-\mathrm{C}}=150.0 \mathrm{~Hz}\right), 62.6\left(\mathrm{~d},{ }^{2} J_{\mathrm{P}-\mathrm{C}}=6.0 \mathrm{~Hz}\right), 61.3\left(\mathrm{~d},{ }^{2} J_{\mathrm{P}-\mathrm{C}}=\right.$ $6.0 \mathrm{~Hz}), 21.3,16.0\left(\mathrm{~d},{ }^{3} J_{\mathrm{P}-\mathrm{C}}=5.8 \mathrm{~Hz}\right), 15.4\left(\mathrm{~d},{ }^{3} \mathrm{~J}_{\mathrm{P}-\mathrm{C}}=5.8 \mathrm{~Hz}\right)$; IR $\left(\mathrm{KBr}, \mathrm{v}_{\max }, \mathrm{cm}^{-1}\right)$ : 3264 (brs, OH), $1233(\mathrm{P}=\mathrm{O})$; Anal. calcd. for $\mathrm{C}_{12} \mathrm{H}_{19} \mathrm{O}_{4} \mathrm{P}: \mathrm{C}, 55.81 ; \mathrm{H}, 7.42$; Found: $\mathrm{C}, 55.77 ; \mathrm{H}, 7.37$.

Diethyl (hydroxy)(4-methoxyphenyl)methylphosphonate (c): white crystals, Yield: $75 \%$, m.p. $119-121{ }^{\circ} \mathrm{C} .{ }^{1} \mathrm{H}$ NMR (CDCl $\left.l_{3}, 400 \mathrm{MHz}\right): \delta$ 7.31-7.01 (m, 4H), 5.19 (s, 1H), $4.63\left(\mathrm{~d},{ }^{2} J_{\mathrm{P}-\mathrm{H}}=10.4 \mathrm{~Hz}, 1 \mathrm{H}\right), 4.20-4.02(\mathrm{~m}, 4 \mathrm{H}), 3.87(\mathrm{~s}, 3 \mathrm{H})$ 1.28-1.19 (m, 6H); ${ }^{13} \mathrm{C} \mathrm{NMR}\left(\mathrm{CDCl}_{3}, 100 \mathrm{MHz}\right): \delta 147.4$, $137.3,122.1,121.3,69.4\left(\mathrm{~d},{ }^{1} J_{\mathrm{P}-\mathrm{C}}=149.0 \mathrm{~Hz}\right), 62.4\left(\mathrm{~d},{ }^{2} J_{\mathrm{P}-\mathrm{C}}=\right.$ $6.1 \mathrm{~Hz}), 62.1\left(\mathrm{~d},{ }^{2} J_{\mathrm{P}-\mathrm{C}}=6.1 \mathrm{~Hz}\right), 57.3,16.0\left(\mathrm{~d},{ }^{3} J_{\mathrm{P}-\mathrm{C}}=6.1 \mathrm{~Hz}\right)$, $15.7\left(\mathrm{~d},{ }^{3} J_{\mathrm{P}-\mathrm{C}}=5.9 \mathrm{~Hz}\right)$; IR $\left(\mathrm{KBr}, v_{\max }, \mathrm{cm}^{-1}\right): 3263$ (brs, OH), $1229(\mathrm{P}=\mathrm{O})$; Anal. calcd. for $\mathrm{C}_{12} \mathrm{H}_{19} \mathrm{O}_{5} \mathrm{P}$ : C, 52.55; H, 6.98; Found: C, 52.45; H, 6.92 .

Diethyl (hydroxy)(3-nitrophenyl)methylphosphonate (d): orange crystals, Yield: $85 \%$, m.p. $83-84{ }^{\circ} \mathrm{C}$. ${ }^{1} \mathrm{H}$ NMR $\left(\mathrm{CDCl}_{3}, 400 \mathrm{MHz}\right.$ ): $\delta 8.18$ (quasi d, $J=8.2 \mathrm{~Hz}, 2 \mathrm{H}$ ), 7.49 (quasi $\mathrm{d}, J=8.2 \mathrm{~Hz}, 2 \mathrm{H}), 5.26(\mathrm{~s}, 1 \mathrm{H}), 4.97\left(\mathrm{~d},{ }^{2} J_{\mathrm{P}-\mathrm{H}}=10.6 \mathrm{~Hz}, 1 \mathrm{H}\right)$, 4.38-4.26 (m, 4H), 1.41-1.28 (m, 6H); ${ }^{13} \mathrm{C} \mathrm{NMR}\left(\mathrm{CDCl}_{3}, 100\right.$ $\mathrm{MHz}): \delta 152.3,148.9,129.7,128.1,127.7,126.3,70.5\left(\mathrm{~d},{ }^{1} J_{\mathrm{P}-\mathrm{C}}\right.$ $=153.0 \mathrm{~Hz}), 64.7\left(\mathrm{~d},{ }^{2} J_{\mathrm{P}-\mathrm{C}}=6.7 \mathrm{~Hz}\right), 63.4\left(\mathrm{~d},{ }^{2} J_{\mathrm{P}-\mathrm{C}}=6.7 \mathrm{~Hz}\right)$, $16.5\left(\mathrm{~d},{ }^{3} J_{\mathrm{P}-\mathrm{C}}=6.0 \mathrm{~Hz}\right), 16.2\left(\mathrm{~d},{ }^{3} J_{\mathrm{P}-\mathrm{C}}=6.0 \mathrm{~Hz}\right)$; IR $\left(\mathrm{KBr}, \mathrm{v}_{\max }\right.$, $\mathrm{cm}^{-1}$ ): 3245 (brs, $\left.\mathrm{OH}\right), 1210(\mathrm{P}=\mathrm{O})$; Anal. calcd. for $\mathrm{C}_{11} \mathrm{H}_{16} \mathrm{NO}_{6} \mathrm{P}$ : C, 45.68; H, 5.58; N, 4.84; Found: C, 45.56; H, 5.55; N, 4.83.

Diethyl (4-chlorophenyl)(hydroxy)methylphosphonate (e): white crystals, Yield: $78 \%$, m.p. $71-73{ }^{\circ} \mathrm{C} .{ }^{1} \mathrm{H}$ NMR $\left(\mathrm{CDCl}_{3}, 400 \mathrm{MHz}\right): \delta$ 7.46-7.23 (m, 4H), 5.15 (s, 1H), 4.79 $\left(\mathrm{d},{ }^{2} J_{\mathrm{P}-\mathrm{H}}=10.9 \mathrm{~Hz}, 1 \mathrm{H}\right), 4.26-3.99(\mathrm{~m}, 4 \mathrm{H}), 1.35-1.24(\mathrm{~m}$, $6 \mathrm{H}) ;{ }^{13} \mathrm{C} \mathrm{NMR}\left(\mathrm{CDCl}_{3}, 100 \mathrm{MHz}\right): \delta 150.4,140.3,129.1$, $128.1,72.4\left(\mathrm{~d},{ }^{1} J_{\mathrm{P}-\mathrm{C}}=159.0 \mathrm{~Hz}\right), 63.79\left(\mathrm{~d},{ }^{2} J_{\mathrm{P}-\mathrm{C}}=6.8 \mathrm{~Hz}\right)$, $16.9\left(\mathrm{~d},{ }^{3} J_{\mathrm{P}-\mathrm{C}}=5.8 \mathrm{~Hz}\right), 16.4\left(\mathrm{~d},{ }^{3} J_{\mathrm{P}-\mathrm{C}}=5.8 \mathrm{~Hz}\right) ; \mathrm{IR}\left(\mathrm{KBr}, \mathrm{v}_{\max }\right.$, $\left.\mathrm{cm}^{-1}\right)$ : 3215 (brs, $\left.\mathrm{OH}\right), 1236(\mathrm{P}=\mathrm{O})$; Anal. calcd. for $\mathrm{C}_{11} \mathrm{H}_{16} \mathrm{O}_{4} \mathrm{PCl}$ : C, 47.41; H, 5.79; Found: C, 47.40; H, 5.66.
Diethyl (hydroxy)(4-nitrophenyl)methylphosphonate (f): orange crystals, Yield: $83 \%$, m.p. $83-85^{\circ} \mathrm{C} .{ }^{1} \mathrm{H}$ NMR $\left(\mathrm{CDCl}_{3}, 400 \mathrm{MHz}\right): \delta 8.20(\mathrm{~d}, J=8.2 \mathrm{~Hz}, 2 \mathrm{H}), 7.48(\mathrm{~d}, J=8.2$ $\mathrm{Hz}, 2 \mathrm{H}), 5.28(\mathrm{~s}, 1 \mathrm{H}), 4.99\left(\mathrm{~d},{ }^{2} J_{\mathrm{P}-\mathrm{H}}=10.6 \mathrm{~Hz}, 1 \mathrm{H}\right), 4.39-4.25$ $(\mathrm{m}, 4 \mathrm{H}), 1.40-1.29(\mathrm{~m}, 6 \mathrm{H}) ;{ }^{13} \mathrm{C} \mathrm{NMR}\left(\mathrm{CDCl}_{3}, 100 \mathrm{MHz}\right): \delta$ $152.4,148.8,128.2,127.9,70.8\left(\mathrm{~d},{ }^{1} J_{\mathrm{P}-\mathrm{C}}=153.0 \mathrm{~Hz}\right), 64.9(\mathrm{~d}$, $\left.{ }^{2} J_{\mathrm{P}-\mathrm{C}}=6.7 \mathrm{~Hz}\right), 63.5\left(\mathrm{~d},{ }^{2} J_{\mathrm{P}-\mathrm{C}}=6.7 \mathrm{~Hz}\right), 16.9\left(\mathrm{~d},{ }^{3} J_{\mathrm{P}-\mathrm{C}}=6.0 \mathrm{~Hz}\right)$, $16.5\left(\mathrm{~d},{ }^{3} J_{\mathrm{P}-\mathrm{C}}=6.0 \mathrm{~Hz}\right)$; IR $\left(\mathrm{KBr}, v_{\max }, \mathrm{cm}^{-1}\right): 3240$ (brs, OH), $1210(\mathrm{P}=\mathrm{O})$; Anal. calcd. for $\mathrm{C}_{11} \mathrm{H}_{16} \mathrm{NO}_{6} \mathrm{P}: \mathrm{C}, 45.68$; H, 5.58; N, 4.84; Found: C, 45.57; H, 5.57; N, 4.81.

\section{RESULTS AND DISCUSSION}

The reaction was optimized using $\mathrm{C}_{6} \mathrm{H}_{5} \mathrm{CHO}$ (3 mmol) as the substrate. Different parameters such as the molar ratio of $\mathrm{C}_{6} \mathrm{H}_{5} \mathrm{CHO} /$ diethyl phosphite $/ \mathrm{Na}_{2} \mathrm{CO}_{3}$ and reaction time were studied (Table-1). When the molar ratio of $\mathrm{C}_{6} \mathrm{H}_{5} \mathrm{CHO} / \mathrm{C}_{4} \mathrm{H}_{11} \mathrm{PO}_{3} /$ $\mathrm{Na}_{2} \mathrm{CO}_{3}$ was 1:1:0.5, the yield of oxime was $70 \%$. Increasing the molar rate to $1: 1: 1$. the yield increase to $82 \%$. When the molar ratio increased to $1: 1: 1.5$, the yield increase to $83 \%$. Further increasing the molar ratio, the yield did not increased. When increased the amount of diethyl phosphite to $1: 1.2: 1$, $1: 1.4: 1$, the yield of the $\alpha$-hydroxyphosphonates was 78 and $81 \%$, respectively. It seemed that the excess of diethyl phosphite had no pronounced effect to the reaction.

\begin{tabular}{cccc}
\multicolumn{4}{c}{ TABLE-1 } \\
\multicolumn{4}{c}{ EFFECT OF THE REACTION CONDITIONS OF } \\
$\alpha$-HYDROXYPHOSPHONATES UNDER GRINDING \\
\hline Entry & Molar ratio of $\mathrm{C}_{6} \mathrm{H}_{5} \mathrm{CHO}$ & Grinding & Yield \\
& /diethyl phosphite $/ \mathrm{Na}_{2} \mathrm{CO}_{3}$ & Time (min) & $(\%)$ \\
\hline $\mathbf{1}$ & $1: 1: 0.5$ & 12 & 70 \\
$\mathbf{2}$ & $1: 1: 1$ & 10 & 82 \\
$\mathbf{3}$ & $1: 1: 1.5$ & 10 & 83 \\
$\mathbf{4}$ & $1: 1: 2$ & 10 & 82 \\
$\mathbf{5}$ & $1: 1.2: 1$ & 14 & 78 \\
$\mathbf{6}$ & $1: 1.4: 1$ & 14 & 81 \\
$\mathbf{7}$ & $1: 1: 0$ & a night & 0 \\
\hline
\end{tabular}

In the absence of $\mathrm{Na}_{2} \mathrm{CO}_{3}$, the mixture was ground for 10 min and kept for a night, the yield of the corresponding $\alpha$ hydroxyphosphonates was $0 \%$. While in the presence of $\mathrm{Na}_{2} \mathrm{CO}_{3}$, the yield of the $\alpha$-hydroxyphosphonates was $82 \%$. It indicated that $\mathrm{Na}_{2} \mathrm{CO}_{3}$ was necessary to the reaction.

On the basis of these results, the optimized reaction conditions we chose were: aldehyde ( $3 \mathrm{mmol}$ ), diethyl phosphite (3 mmol) and $\mathrm{Na}_{2} \mathrm{CO}_{3}(3 \mathrm{mmol})$. Using this reaction system, we performed a series of experiments for $\alpha$-hydroxyphosphonates of aldehydes by grinding. The results were summarized in Table-2.

\begin{tabular}{|c|c|c|c|c|}
\hline \multicolumn{5}{|c|}{$\begin{array}{c}\text { TABLE-2 } \\
\text { SYNTHESIS OF } \alpha \text {-HYDROXYPHOSPHONATES IN GRINDING METHOD }\end{array}$} \\
\hline Enty & $\mathrm{R}$ & m.p. $\left({ }^{\circ} \mathrm{C}\right)$ & Colour & Yield (\%) \\
\hline $\mathbf{a}$ & $\mathrm{Ph}$ & $83-85$ & White crystals & 82 \\
\hline $\mathbf{b}$ & $p-\mathrm{CH}_{3} \mathrm{Ph}$ & $98-100$ & White crystals & 76 \\
\hline c & $p-\mathrm{CH}_{3} \mathrm{OPh}$ & $119-121$ & White crystals & 75 \\
\hline d & $m-\mathrm{NO}_{2} \mathrm{Ph}$ & $83-84$ & Orange crystals & 85 \\
\hline $\mathbf{e}$ & $p-\mathrm{ClPh}$ & $71-73$ & White crystals & 78 \\
\hline f & $p-\mathrm{NO}_{2} \mathrm{Ph}$ & $83-85$ & Orange crystals & 83 \\
\hline
\end{tabular}


As shown in Table-2, some aldehydes via condensation can give $\alpha$-hydroxyphosphonates in good yields in free solvent under grinding. Substitution on aromatic aldehyde played a crucial role in governing the product yield as it can be seen from the Table-2. Electron-withdrawing group on benzaldehyde gave a good yield (Table-2, entry d), whereas electrondonating group on benzaldehyde gave a lower yield (Table-1, entry b) in comparison with benzaldehyde.

\section{Conclusion}

In conclusion, a general and highly efficient procedure for the preparation of $\alpha$-hydroxyphosphonates catalyzed by $\mathrm{Na}_{2} \mathrm{CO}_{3}$ with grinding method is described. In addition, the procedure offers several advantages including high yields, cleaner reactions, operational simplicity, minimal environmental impact which makes it a useful and attractive process for the synthesis of these compounds. Moreover, it is possible to apply the tenets of green chemistry to the generation of biologically interesting products with grinding method which are less expensive and less toxic than those with organic solvents.

\section{ACKNOWLEDGEMENTS}

The authors are grateful to China National Science Foundation of P.R. China (No: 21162008) for financial supports.

\section{REFERENCES}

1. J.G. Dingwall, U.K. Patent 1542938 (1979).

2. R. Engel, Chem. Rev., 77, 349 (1977).

3. B. Iorga, F. Eymery and P. Savignac, Tetrahedron, 55, 2671 (1999).

4. G.A. Olah and A.H. Wu, J. Org. Chem., 56, 902 (1991).

5. R.U. Pokalwar, R.V. Hangarge, P.V. Maske and M.S. Shingare, ARKIVOC, 196 (2006)

6. Y. Ishiguri, E.P. Patent, 82-301905 (1982).

7. D.V. Patel, K. Rielly-Gauvin and D.E. Ryono, Tetrahedron Lett., 31, 5587 (1990).

8. A.R. Sardarian and B. Kaboudin, Synth. Commun., 27, 543 (1997).

9. V. Thottempudi and K.-H. Chung, Bull. Korean Chem. Soc., 29, 1781 (2008).

10. X. Zhou, X. Liu, X. Yang, D. Shang, J. Xin and X. Feng, Angew. Chem. Int. Ed., 120, 398 (2008).

11. A.A. Smaardijk, S. Noorda, F. van Bolhuis and H. Wynberg, Tetrahedron Lett., 26, 493 (1985).

12. V.J. Blazis, K.J. Koeller and C.D. Spilling, J. Org. Chem., 60, 931 (1995).

13. T. Yokomatsu, T. Yamagishi and S. Shibuya, J. Chem. Soc. Perkin Trans. I, 1527 (1997).

14. N. Azizi and M.R. Saidi, Phosphorus Sulfur Silicon Rel. Elem., 178, 1255 (2003).

15. (a) K. Tanaka and F. Toda, Chem. Rev., 100, 1025 (2000); (b) Z.J. Ren, W.G. Cao and W.Q. Tong, Synth. Commun., 32, 3475 (2002); (c) F. Toda, K. Tanaka and K. Hamai, J. Chem. Soc. Perkin Trans., 3207 (1990); (d) F. Toda, T. Suzuki and S. Higa, J. Chem. Soc. Perkin Trans., 3251 (1998); (e) K. Tanaka, S. Kishigami and F. Toda, J. Org. Chem., 56, 4333 (1991); (f) F. Toda, K. Kiyoshige and M. Yagi, Angew. Chem. Int. Ed. Engl., 28, 320 (1989); (g) H. Hagiwara, S. Obtrubo and M. Kato, Mol. Cryst. Liq. Cryst., 279, 291 (1996). 